\title{
Salt enrichment and dynamics in the interface of supercooled aqueous droplets
}

\author{
Victor Kwan, ${ }^{\dagger}$ Shoubhik R Maiti, ${ }^{\dagger, \dagger}$ Ivan Saika-Voivod, ${ }^{\top}$ and Styliani Consta ${ }^{* \dagger}$ \\ $\dagger$ Department of Chemistry, The University of Western Ontario, London, Ontario, Canada \\ N6A $5 B 7$ \\ $\ddagger$ Department of Chemistry, The University of Sheffield, Sheffield S3 7HF, United Kingdom \\ 【 Department of Physics and Physical Oceanography, Memorial University of \\ Newfoundland, Canada, A1B $3 X^{r}$ \\ E-mail: sconstas@uwo.ca
}

\section{Abstract}

The interconversion reaction of $\mathrm{NaCl}$ between the contact-ion pair (CIP) and the solventseparated ion pair (SSIP) as well as the free-ion state in cold droplets have not been yet investigated. We report direct computational evidence that the lower the temperature the closer to the surface the ion interconversion reaction takes place. In supercooled droplets the enrichment of the subsurface in salt becomes more evident. The stability of the SSIP relative to the CIP increases as the ion-pairing is transferred toward the droplet's outer layers. In the free ion form, the number density of $\mathrm{Cl}^{-}$in nanodroplets shows a broad maximum in the interior in addition to the well-known maximum in the surface. In the study of the reaction dynamics, we find a weak coupling between the interionic $\mathrm{NaCl}$ distance reaction coordinate and the solvent degrees of freedom, which contrasts the diffusive crossing of the free energy barrier found in bulk solution modeling. The $\mathrm{H}_{2} \mathrm{O}$ selfdiffusion coefficient is found to be at least an order of magnitude larger than that in the bulk solution. This fast diffusion decreases the coupling of the solvent with the interionic distance reaction coordinate. We propose to exploit the enhanced surface ion concentration at low temperature to eliminate salts from droplets in native mass spectrometry ionization methods.
Reduction of adduct formation between macromolecules and salt may open up the possibility to directly ionize a biological sample from a physiological solution in experiments.

\section{Introduction}

Cold aqueous droplets containing ion pairs play an important role in atmospheric chemistry and technology. In particular, they participate in thundercloud processes in the upper troposphere, where the temperature may be as low as $220 \mathrm{~K}$. In technology, they are used in cryo-preservation, ${ }^{1,2}$ and potentially they can be used in geo- and climate-engineering. Cold droplets also show particular physical properties. A notable example is the preservation within nanodroplets of the supercooled liquid state, in contrast to the bulk, where practically unavoidable crystallization prevents the liquid from existing in the temperature range of $T=150 \mathrm{~K}$ to $230 \mathrm{~K}$ at $P=1$ atm of pressure. ${ }^{3-16}$ In a broader context, the distinct physical chemistry in small-volume cold systems has been demonstrated by the formation of new ice structures that cannot been seen in the bulk. ${ }^{3-14,17}$

Cold aqueous nanodroplets have a surprisingly complex internal structure that varies with temperature and size, as revealed by computer simulations of the TIP4P/2005 model of 
water. ${ }^{18}$ While the interior $P$ of small droplets reaches over $100 \mathrm{MPa}$ on account of a surface tension that increases with decreasing $T$, two other factors conspire to create a heterogeneous environment: densification of the liquid at the vapour interface, and a bulk-like interior that reflects the bulk behavior of water, including a tetrahedral network that expands and becomes more compressible with decreasing $T$. By $T=220 \mathrm{~K}$, the outer water layers can become significantly denser compared to the interior of the droplet. The interior core density continues to follow bulk values for the given $T$ and interior $P$. For droplets with number of molecules $N \geq 360$, the droplet interior exhibits a temperature of maximum density below which the density decreases, creating a density contrast between the lower density core and higher density (sub)surface, a contrast that sharpens with decreasing $T$. For $T \leq 200$, the density differences between core and subsurface exceeds 5\%. The above scenario means that simulated supercooled droplets (for $N \geq 200$ ) exhibit structural heterogeneity manifested by three radial regions: low-density interior, highdensity subsurface (thickness $\approx 0.7-0.8 \mathrm{~nm}$ ) and surface (thickness $\approx 0.3 \mathrm{~nm}$ ). These regions are depicted in Fig. 1. This structure provides an interesting chemical environment that contrasts that of a simpler nanodroplet at ambient conditions, where the density is approximately constant, showing a decrease (mainly due to shape fluctuations) only at the liquidvapor interface.

The study of the distinct chemical reactivity within supercooled droplets, ${ }^{2}$ and more generally in cold droplets is still in its infancy. Here, we model the $\mathrm{NaCl}$ ion-pairing, one of the fundamental reactions in chemistry, in mesoscopic clusters in the temperature range of $200 \mathrm{~K}$ $300 \mathrm{~K}$. The mesoscopic clusters are also called nanodroplets.

Experiments and computations of $\mathrm{NaCl}$ ionpairs in clusters have been mainly performed in systems composed of up to a few tens of $\mathrm{H}_{2} \mathrm{O}$ molecules. ${ }^{20-30}$ Fourier transform microwave spectroscopy ${ }^{24}$ has shown that the distance between the ions in a $\mathrm{NaCl}$ pair increases by gradually adding up to $3 \mathrm{H}_{2} \mathrm{O}$ molecules and

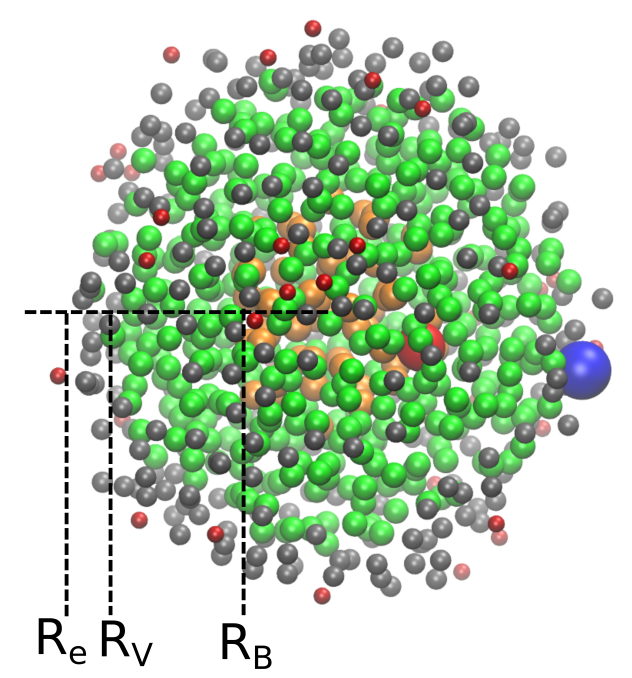

Figure 1: Typical snapshot of a supercooled droplet composed of $N=776 \mathrm{H}_{2} \mathrm{O}$ molecules and a sole $\mathrm{NaCl}$ ion pair. Only the $\mathrm{O}$ sites are depicted in the colored shells, the $\mathrm{Na}^{+}$is depicted by the large red-colored sphere, and the $\mathrm{Cl}^{-}$by the blue-colored sphere. Three regions are distinguished where the $\mathrm{H}_{2} \mathrm{O}$ molecules are organized differently: (a) A bulk-like interior (orange-colored) that extends from the droplet center of mass (COM) to a distance $r=R_{B}$. This region is characterized by a lower density and a better formed tetrahedral network than the outer layers. (b) The subsurface region $R_{B}<r<R_{V}$ (green-colored), that is characterized by a higher density, and lower degree of tetrahedrality than the interior. (c) The surface region $R_{V}<r<R_{e}$ (grey-colored), where $R_{e}$ is the equimolar radius. The determination of $R_{B}$ and $R_{V}$ is presented in Ref. ${ }^{19} \mathrm{~A}$ few $\mathrm{H}_{2} \mathrm{O}$ molecules (O sites are depicted by small redcolored spheres) are found at $r>R_{e}$.

reaches the value of $2.9 \AA$, which is the contaction pair (CIP) distance found in the bulk solution. Anion photoelectron spectroscopy ${ }^{25}$ of clusters comprising $\mathrm{NaCl}$ and up to $12 \mathrm{H}_{2} \mathrm{O}$ molecules found that at 9-12 $\mathrm{H}_{2} \mathrm{O}$ molecules, the CIP and SSIP (solvent-separated ion pair) formations are almost degenerate in energy. In larger clusters, characteristics different from those of minute or macroscopic sizes, such as the large shape fluctuations, prevail and may affect the reaction mechanisms. The experi- 
mental and computational studies of salt ionpairing in larger cluster are more limited. Clusters of 150-400 $\mathrm{H}_{2} \mathrm{O}$ molecules containing $\mathrm{NaCl}$ have been studied by X-ray photoelectron spectroscopy. ${ }^{31,31,32}$

Nanodroplets are characterized by a large surface-to-volume ratio, thus, when they are neutral and sufficiently large, they may have commonalities with the ion-pairing features in planar interfaces. The $\mathrm{NaCl}$ ion pairing in the planar water interface at room temperature has been extensively studied. ${ }^{26,33-41}$ It has been found that the CIP and SSIP are stabilized differently in the interface and in the bulk. There is a high degree of agreement that the ion dissociation is suppressed in the interface ${ }^{33,34,38}$ relative to bulk solution.

The study of the ion pairing in cold systems is more challenging because of the slow dynamics. ${ }^{42,43}$ The ion-pairing mechanism has been only recently explored in the premelting ice surface. ${ }^{44}$ It has been found that the ice interface may play the dominant role in the reaction dynamics and that the dissociation dynamics of water molecules in the premelting layer differ significantly from those in bulk solution.

In this article we use the example of $\mathrm{NaCl}$ in an aqueous nanodroplet to examine via molecular modeling how the water structure and dynamics at cooling, and more evidently at supercooling, affect the ion-pairing. Differently from the simulations of ion pairs in planar interfaces, ${ }^{38}$ where the ions are restricted to remain in the interface, in the droplet simulations the ions are free to explore the entire volume. We hypothesize that the colder the droplet, the more likely is for the CIP to SSIP interconversion reaction to occur in the droplet subsurface and surface. The dynamics in the droplets is characterized by the $\mathrm{H}_{2} \mathrm{O}$ self-diffusion coefficient and the rate constant for the CIP to SSIP transition. As it will be discussed later, the salt enrichment of the interface at low temperature may be exploited to optimize the ionization conditions in mass spectrometry (MS).

\section{Results}

\section{Equilibrium}

To examine how the particular structure of a cold droplet affects the ion-pairing we mainly study systems comprising $N=776 \mathrm{H}_{2} \mathrm{O}$ molecules and a sole $\mathrm{NaCl}$ pair in the temperature range of $200 \mathrm{~K}$ to $300 \mathrm{~K}$. The droplet size is large enough for the results to be transferable to larger droplets. We model the systems with the TIP4P/2005 set of parameters and with the polarizable SWM4-NDP force field. ${ }^{45}$ TIP4P/2005 allows one to model the systems at supercooling, but it lacks the electronic polarization. In previous research it has been found that the SWM4-NDP model yields non-physical water density at low temperature, ${ }^{19,46,47}$ and for this reason we can only study the trends in dynamic and equilibrium properties in the temperature range $T=260 \mathrm{~K}-300 \mathrm{~K}$.

The TIP4P/2005 model shows that the number of transitions between CIP and SSIP as well as between SSIP and the free ions (Fig. S1 in $\mathrm{SI})$ is sufficient to provide good enough statistics to directly calculate free energy differences along the $\mathrm{Na}^{+}-\mathrm{Cl}^{-}$interionic distance. Indicatively, at $T=200 \mathrm{~K}$ there are 7 transitions from CIP to SSIP within $2.8 \mu \mathrm{s}$, and at $T=220 \mathrm{~K}$ 10 transitions within $0.8 \mu \mathrm{s}$ (Fig. S1 in SI). In Fig. 2 (a) (for TIP4P/2005 model) the free energy along the $\mathrm{Na}^{+}-\mathrm{Cl}^{-}$interionic distance at $T=200 \mathrm{~K}$ and $300 \mathrm{~K}$ are shown. The CIP is defined within the basin where the $\mathrm{Na}^{+}-\mathrm{Cl}^{-}$interionic distance is in the range of $2.5 \AA-3.5 \AA$, the SSIP in 3.5 $\AA-5.7 .0 \AA$, and the free ions when they are $>5.7 \AA$ apart. The equilibrium constant for $\mathrm{CIP} \rightleftharpoons \mathrm{SSIP}$ and the free energy differences along the interionic distance are shown in Table 1 . It is found that at $T=300 \mathrm{~K}$ the CIP is stabilized more than at $200 \mathrm{~K}$ and that at $200 \mathrm{~K}$, the free energy difference between CIP and SSIP decreases.

Figure 2 (b) and (c) show the location at which the ion-pairing takes place at $200 \mathrm{~K}$ and $300 \mathrm{~K}$, respectively. The free-ion configurations accounts for $\approx 70 \%$ of the total number. In previous research ${ }^{19}$ we have found that the supercooled droplets with $N>200$ exhibit het- 
Table 1: Free energy differences $\left(\Delta W_{1,2}\right)$ along the $\mathbf{N a}^{+}-\mathrm{Cl}^{-}$distance at various temperatures for TIP4P/2005 and SWM4-NDP models. BT denotes the free energy barrier top along this reaction coordinate. The number in parentheses is $\Delta W_{1,2}$ in units of $k_{B} T$, where $k_{B}$ is the Boltzmann constant. $K_{\text {eq }}$ is the equilibrium constant for CIP $\rightleftharpoons$ SSIP. The statistical error in the free energy estimates is $\pm 0.15 \mathrm{kcal} / \mathrm{mol}$.

\begin{tabular}{|c|c|c|c|c|}
\hline Model & $T(\mathrm{~K})$ & $\begin{array}{c}\Delta W_{1}(\mathrm{kcal} / \mathrm{mol}) \\
\mathrm{CIP} \longrightarrow \mathrm{BT}\end{array}$ & $\begin{array}{c}\Delta W_{2}(\mathrm{kcal} / \mathrm{mol}) \\
\mathrm{SSIP} \longrightarrow \mathrm{BT}\end{array}$ & $K_{\text {eq }}$ \\
\hline TIP4P/2005 & 300 & $2.8(4.7)$ & $1.8(3.0)$ & 0.74 \\
& 260 & $2.6(5.1)$ & $2.1(4.0)$ & 1.09 \\
& 240 & $2.8(5.8)$ & $2.5(5.2)$ & 1.52 \\
& 210 & $2.3(5.4)$ & $2.1(5.0)$ & 1.92 \\
& 200 & $2.5(6.3)$ & $2.4(6.0)$ & 1.77 \\
\hline SWM4-NDP & 300 & $1.4(2.35)$ & $2.2(3.7)$ & 10.2 \\
& 280 & $1.3(2.34)$ & $2.3(4.1)$ & 19.9 \\
& 260 & $1.3(2.52)$ & $2.3(4.5)$ & 22.5 \\
\hline
\end{tabular}

erogeneity that is manifested by three regions: a bulk-like interior, a subsurface, and a surface (Fig 1). In $N=776$ the distances from the droplet's COM that delimit these regions are $R_{\mathrm{B}}=7.4 \AA, R_{\mathrm{V}}=14.8 \AA$, and $R_{\mathrm{e}}=17.7 \AA$ (where $R_{\mathrm{e}}$ denotes the equimolar radius), respectively. At $200 \mathrm{~K}$, the CIP and SSIP show a distinct propensity to be in the middle of the subsurface. In contrast, at $300 \mathrm{~K}$, the probability of formation of CIP, SSIP is significant throughout the volume of the droplet but with a slightly higher probability in the subsurface. We define the angle between the dipole moment of the CIP and SSIP (dipole moment is defined from the negative site to the positive site) and the vector defined from the droplet's COM to the ion-pair COM. At $T=300 \mathrm{~K}$ the orientation of the CIP spans all the angles, but at $200 \mathrm{~K}$, the cosine of the angle is peaks in the range $0.0-0.2$, which corresponds to an angle of $90^{\circ}-78^{\circ}$ (Fig. S2 in SI). The orientation of the $\mathrm{NaCl}$ is clearly more pronounced within the SWM4-NDP model (Fig. S2 in SI). The CIP orientation indicates a slight preference of the $\mathrm{Cl}^{-}$site toward the droplet's surface. Even though Fig. 2 only shows the CIP $\rightleftharpoons$ SSIP equilibrium at two significantly different temperatures, the propensity of the ions toward the subsurface increases gradually as the temperature decreases as shown in Fig. S3 and Fig. S4 in SI. Specifically, we find that TIP4P/2005 at $200 \mathrm{~K}$ expels $\mathrm{Na}^{+}$stronger than $\mathrm{Cl}^{-}$(Fig. $\mathrm{S} 4$ (d) in
SI).

We think that the increased expulsion of the ions in the subsurface as temperature decreases is due to an improved H-bonded coordinated network in the droplet interior. The differences in the H-bonded network in the interior and subsurface in supercooled droplets have been presented in our previous research. ${ }^{19}$ At supercooling, both the CIP and SSIP are mainly formed in the outer portion of the subsurface, where the dielectric constant is expected to be higher than that at $300 \mathrm{~K}$. The higher dielectric constant at lower temperature may lead to a decrease in the free energy difference between the CIP and SSIP, which is consistent with the free energy profile at $200 \mathrm{~K}$ shown in Fig 2 (a).

Figure 3 (a) shows the free energy of the interconversion between CIP and SSIP as a function of the $\mathrm{NaCl}$ interionic distance using SWM4NDP model. The $K_{e q}$ values and the free energy differences are shown in Table 1 . The freeion configurations account for $\approx 85-87 \%$ of the total production run. Figure 3 (b) and (c) show that the CIP is formed near the surface at $300 \mathrm{~K}$ and $260 \mathrm{~K}$. The probability to encounter the ions in the interior and subsurface decreases considerably relative to the TIP4P/2005. The polarizable model finds the $\mathrm{Cl}^{-}$to be nearer to the vapor-liquid interface than $\mathrm{Na}^{+}$. The closer proximity of $\mathrm{Cl}^{-}$to the surface is in agreement with previous studies ${ }^{22,26,48-50}$ that have been performed at room temperature. The temper- 


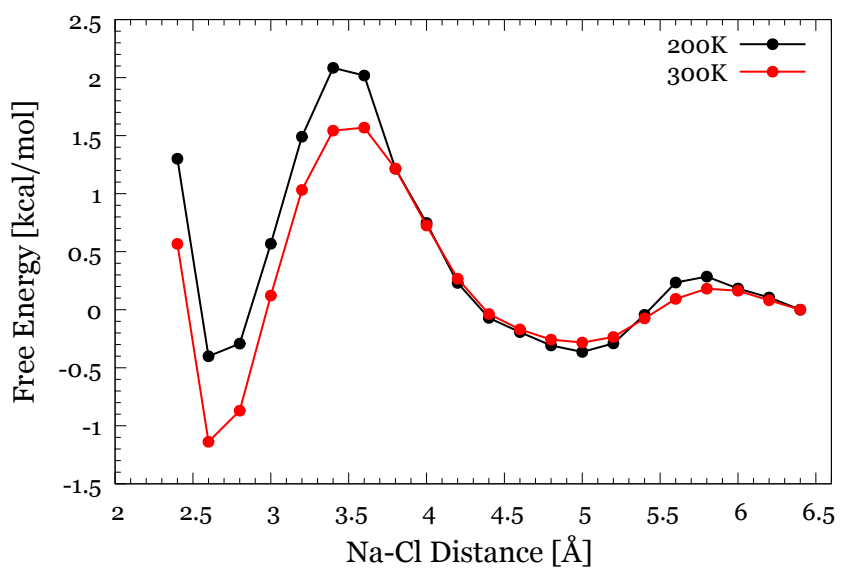

(a)

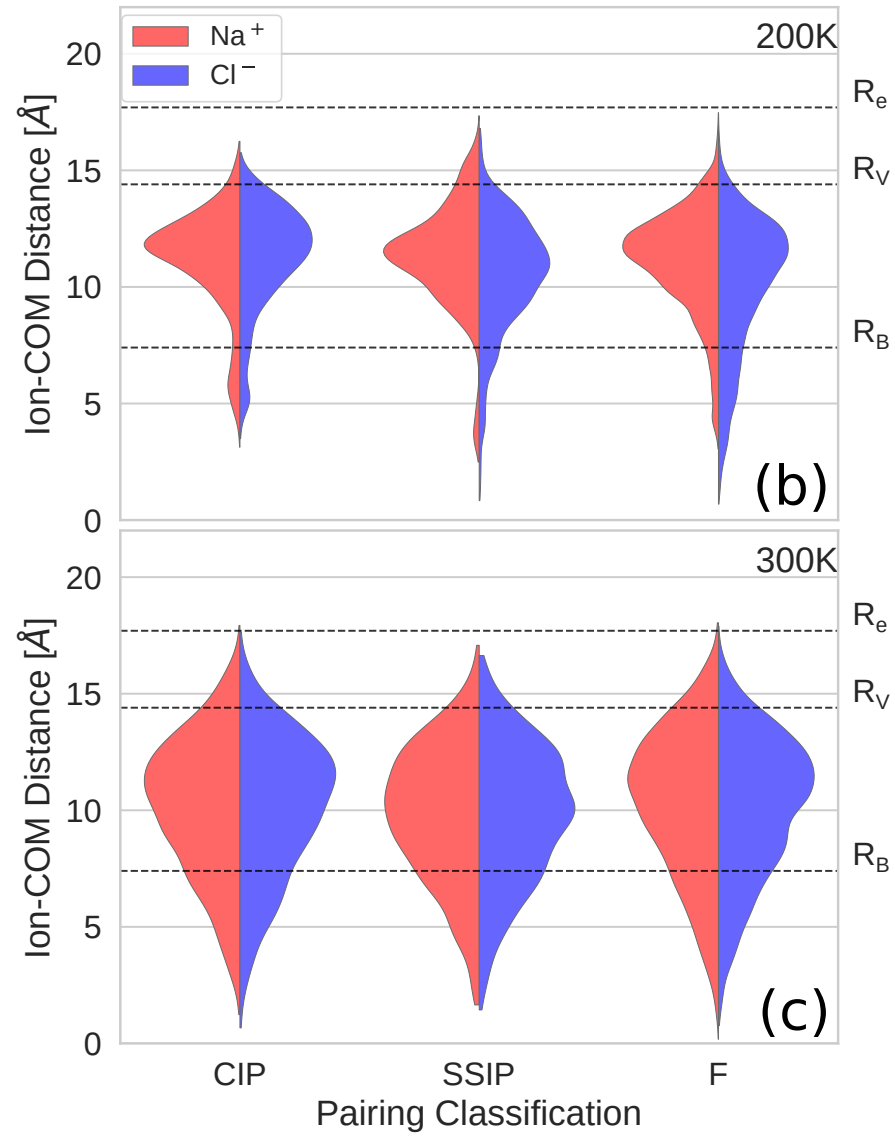

Figure 2: (a) Free energy for CIP $\rightleftharpoons$ SSIP interconversion reaction at $200 \mathrm{~K}$ and $300 \mathrm{~K}$ along the $\mathrm{Na}^{+}{ }_{-} \mathrm{Cl}^{-}$interionic distance. TIP4P/2005 model is used. (b) Distribution of $\mathrm{Na}^{+}$(red) and $\mathrm{Cl}^{-}$(blue) sites in the CIP, SSIP and Free ions (F) basins at $200 \mathrm{~K}$ as a function of the distance from the droplet's COM (y-axis) (the profiles are not normalized by the volume of the spherical shell). (c) Same as (b) but at $300 \mathrm{~K}$.

ature effect, that has not been studied previously, shows that the lower the temperature (a)

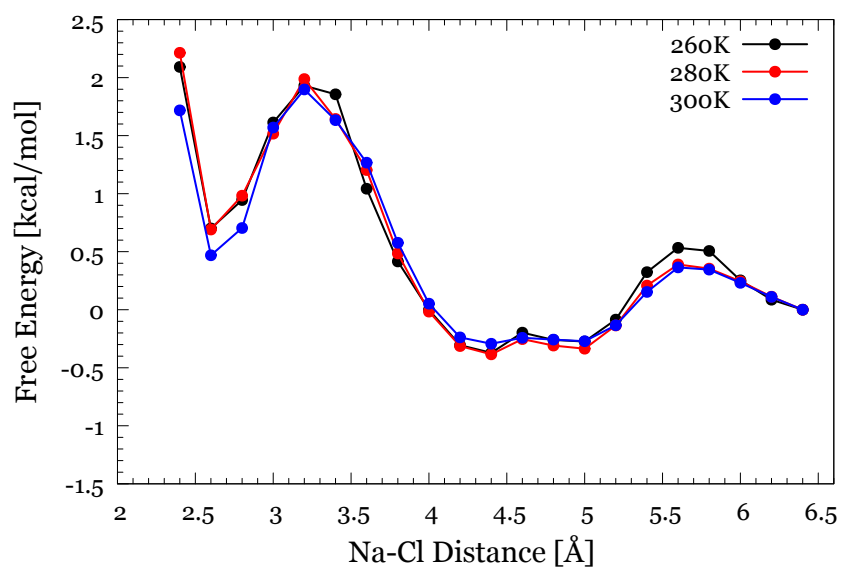

(a)

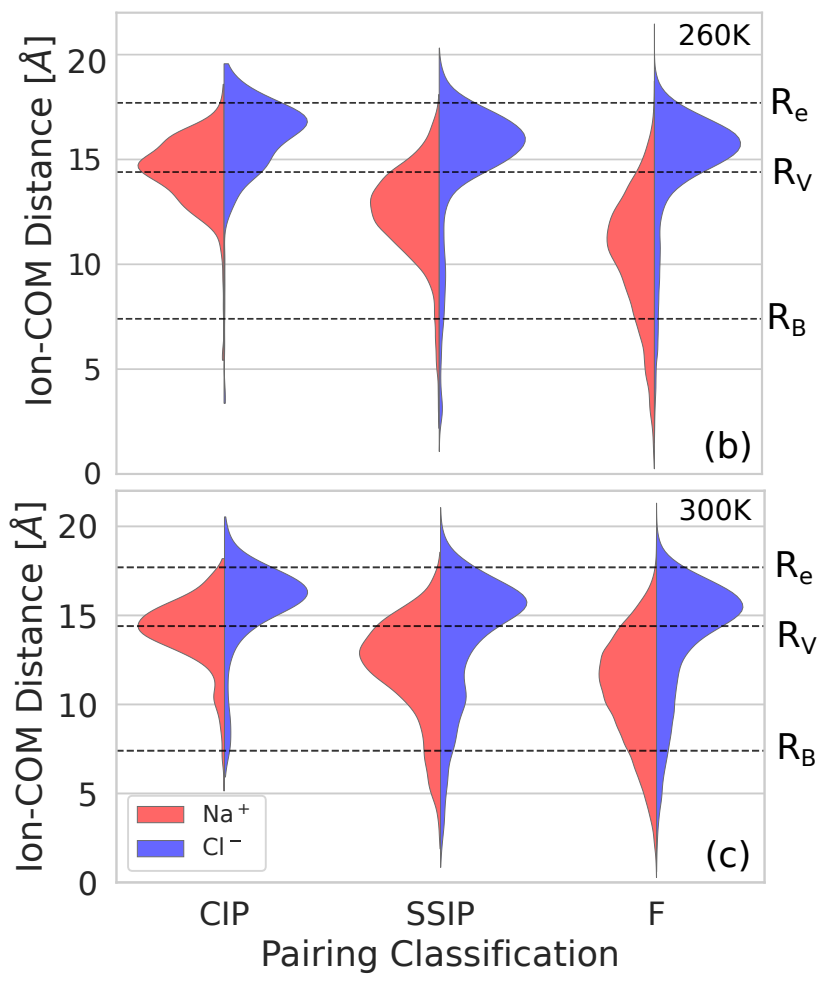

Figure 3: (a) Same as Fig. 2 (a) but for the SWM4-NDP polarizable model at various temperatures. (b) and (c) same as Fig. 2 (b) and (c), respectively, but for SWM4-NDP.

the higher the $\mathrm{Cl}^{-}$probability density in the surface (see also Fig. S5 and Fig. S6 in SI), (b) the free energy differences between the CIP and SSIP and the BT (barrier top) are not very sensitive to the temperature. We attribute this lack of sensitivity to the fact that the polarizable model predicts the most probable location of the ions near the surface in the temperature range $260 \mathrm{~K}-300 \mathrm{~K}$. The location does 


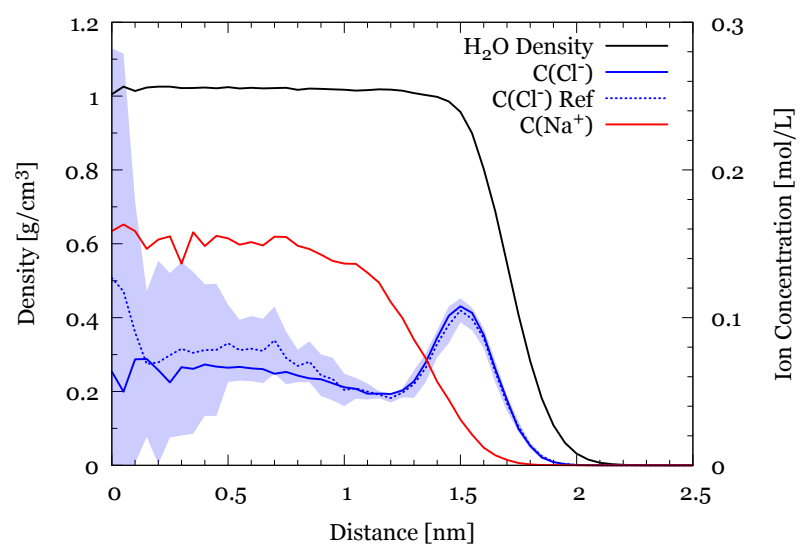

Figure 4: Probability density profiles of $\mathrm{Na}^{+}$ and $\mathrm{Cl}^{-}$sites (measured in units of concentration in the right $\mathrm{y}$-axis), and $\mathrm{H}_{2} \mathrm{O}$ density profiles (measured in units of $\mathrm{g} / \mathrm{cm}^{-3}$ ) in the left y-axis) at $T=300 \mathrm{~K}$ in droplets comprising $N=776 \mathrm{H}_{2} \mathrm{O}$ molecules and a sole $\mathrm{NaCl}$ pair. For reference, the probability density of a sole $\mathrm{Cl}^{-}$in $N=776$ is included. The SWM4-NDP polarizable model is used.

not change significantly with temperature. We note that we have also performed simulations with different values of the Langevin damping (Fig. S7 in SI), and all the free energy profiles are in agreement.

Here, we point out a significant difference in the relative stability of CIP and SSIP between the present study and that of Dang et al. using a polarizable model when the pair is confined in the Gibbs dividing surface of a planar interface. ${ }^{38}$ In the latter case, it is found that the CIP is formed with a higher probability than the SSIP. We think that when the pair is confined in the Gibbs dividing surface both $\mathrm{Na}^{+}$ and $\mathrm{Cl}^{-}$are partially solvated, which promotes pairing due to the low dielectric constant environment. In our systems, the $\mathrm{Cl}^{-}$lies on the surface, but the $\mathrm{Na}^{+}$is well solvated in the borderline of the subsurface and surface or within the subsurface. The likelihood of the CIP formation relative to the SSIP decreases because of the particular requirements for the orientation of the $\mathrm{H}_{2} \mathrm{O}$ molecules near the interface.

The SSIP basin (Fig. 3 (a)) is wide and shows a fine structure of two small local minima. This structure is consistent throughout the temperature range $260 \mathrm{~K}-300 \mathrm{~K}$ and agrees very well with the structure observed in the PMF reported by Dang et al. using a different polarizable model. ${ }^{38}$ Possibly the structure seen in the SSIP basin is due to a different orientation of the bridging $\mathrm{H}_{2} \mathrm{O}$ molecules. As we are going to see later, this structure is consistent even in the presence of four $\mathrm{NaCl}$ pairs.

In summary, even though the polarizable and the non-polarizable force fields show different location of the ion-pairing at room temperature, both models indicate that the lower the temperature the farther from the droplet's COM the ion pairing takes place. The models are also in agreement that the stability of the SSIP relative to the CIP increases as the ionpairing is transferred toward the droplet's outer layers and that when the CIP is formed the $\mathrm{Cl}^{-}$ is found more in the exterior than when it is in the SSIP or free-ion form.

In order to have a closer look to the freeion state we compare the $\mathrm{Na}^{+}$and $\mathrm{Cl}^{-}$number density with that of the sole ions using the SWM4-NDP model at $300 \mathrm{~K}$ as shown in Fig. 4. The free ion-state is the most likely state of the ion pair $(87 \%$ of the total number of configurations). We find that the $\mathrm{Na}^{+}$number density, is higher in the interior in agreement with previous research. ${ }^{19,51,52}$ We computed the number density of $\mathrm{Cl}^{-}$at $T=300 \mathrm{~K}$ as a function of the distance from the droplet's COM. The number density shows two broad maxima. The basin of the one maximum near the surface extends from the subsurface to the surface region (1.2 $\AA-1.9 \AA)$ and corresponds to a free energy minimum of $1.7 k_{B} T$ measured from the barrier top, which is located at $1.2 \AA$. This free energy estimate is in good agreement with the value of $\approx 1.0 k_{B} T$ reported by Caleman et al. ${ }^{51}$ at $T=293.15 \mathrm{~K}$, using the same polarizable model as we do, in a cluster of radius equal to $1.1 \mathrm{~nm}$, which corresponds to $N \approx 145-150 \mathrm{H}_{2} \mathrm{O}$ molecules. The value of this free energy as a function of the distance from the droplet's COM is affected by the shape fluctuations. The smaller the droplet, the larger the effect. Our free energy estimate (and the Ref. ${ }^{51}$ ) includes the contribution from the pure entropic effect due to the spherical geometry. If the free energy due to the geometric effect is subtracted, 
then the effect due to the ion- $\mathrm{H}_{2} \mathrm{O}$ interactions equals $0.90 k_{B} T$. The $N=776$ droplet showed another broad maximum at $0.7 \AA$. The basin that is associated with it is in the range of 0.2 $\AA-1.1 \AA$. It is likely, that the basin extends up to the droplet's COM, but it is harder to distinguish that because of the larger statistical uncertainty near the center. To our knowledge the second maximum has not been previously reported in the literature. The interior basin $(0.2$ $\AA-1.2 \AA$ ) corresponds to a free energy that is more shallow than that on the surface, but it is wider, which indicates a substantial probability to encounter the $\mathrm{Cl}^{-}$in the interior. The equilibrium constant estimated directly by the number of configurations in the exterior basin over the interior is 3.2 , however, if we estimate the ratio of probability densities in the two basins, then the value is 1.1. The presence of $\mathrm{Na}^{+}$increases slightly the residence time of the $\mathrm{Cl}^{-}$on the surface, which decreases the number density of the $\mathrm{Cl}^{-}$in the interior.

To analyse the retention of salt in the droplet interior at supercooled droplets, we also examined droplets comprised $776 \mathrm{H}_{2} \mathrm{O}$ molecules and four $\mathrm{NaCl}$ pairs, using the SWM4-NDP at $260 \mathrm{~K}$ and $300 \mathrm{~K}$ and TIP4P/2005 at $300 \mathrm{~K}$ down to $200 \mathrm{~K}$. The probability density profiles (Fig.S8 and Fig.S9 in SI) and free energy profiles as a function of the interionic distance are shown in Fig. S10 and Fig. S11 in SI. The striking feature at lower temperature is the increased $\mathrm{Na}^{+}$and $\mathrm{Cl}^{-}$probability density in the subsurface (distance from the COM > $1.2 \mathrm{~nm}$ ) relative to that at $300 \mathrm{~K}$. The distance $1.2 \mathrm{~nm}$ separates two basins. For SWM4-NDP the equilibrium constant as a ratio of configurations in the outer basin over the inner basin for $\mathrm{Cl}^{-}$is 12.2 at $260 \mathrm{~K}$ vs 3.3 at $300 \mathrm{~K}$. This ratio for $\mathrm{Na}^{+}$is very similar $\approx 0.84$ at both temperatures. The enrichment of the subsurface in ions at lower temperature is attributed to the expulsion of the ions from the bulk-like interior due to a better coordinated H-bonded network. The reduced shape fluctuations may contribute to this increase as well. We find here that the internal H-bonded network is maintained even at the higher concentration of $\mathrm{NaCl}$. It is interesting to note that Fig. S8 in SI may create the impression that at room temperature the ions are deeper in the interior measured from the surface than at lower temperature. The depth of the ions from the surface is the same at the various temperatures. The visual misconception arises from the fact that the distance from the COM order parameter masks the droplet's shape fluctuations. Comparison of the location of $\mathrm{Na}^{+}$and $\mathrm{Cl}^{-}$between the single pair in the droplet and the multiple pairs, shows that in the supersaturated droplet the ions have higher propensity toward the exterior. The free energy differences of CIP and SSIP (Fig. S10 and Fig. S11 in SI) are in excellent agreement with those for the single $\mathrm{NaCl}$ (Fig. 3 (a) and Table 1), which verifies the convergence of the runs.

It is interesting to note that for several ions in TIP4P/2005 we have consistently found that near $R_{B}$ the probability density shows a minimum. This unfavourable region indicates the presence of an energetic barrier, arising from the different quality of the H-bonded network, in the crossing of the ion from the subsurface to the interior.

Finally, we can examine the effect of the lower temperature in the location of the multiple ions in charged droplets, where ions of the same sign are in excess. We have studied droplets composed of $3000 \mathrm{H}_{2} \mathrm{O}$ molecules and $24 \mathrm{Na}^{+}-8 \mathrm{Cl}^{-}$ ions or $24 \mathrm{Cl}^{-}-8 \mathrm{Na}^{+}$ions at $T=240 \mathrm{~K}$ and $300 \mathrm{~K}$ using TIP4P/2005. The data and analysis are presented in Sec. S8 in SI. The key idea of the surface enrichment in salts also appears in the highly charged droplets. TIP4P/2005 shows the trend for the enrichment, and we expect that a polarizable model will show an even higher enhancement of the surface concentration. In native mass spectrometry (MS) droplets are heated up in order to quickly evaporate but in this process ions may form undesirable adducts with the analytes. We propose that by initial cooling a droplet instead of heating, or by alternating heating and cooling the ions may accumulate near the surface. Possibly, bombardment with other molecules may assist in the detachment of solvated ions or their salt complexes from the surface. 


\section{Dynamics}

The dynamics of the ion-pair interconversion reaction may be affected by the self-diffusion of the $\mathrm{H}_{2} \mathrm{O}$ molecules. We compute the self-diffusion coefficient using the fluctuationdissipation theorem where we combine a correlation function formalism with the macroscopic description of the diffusion within a spherical cavity. The details of the method are described in the Methods Section. The diffusion coefficient as a function of temperature is shown in Table 2 and Fig. 5. An example of the decay of $\left\langle\delta J_{k, l, m}^{\star}(0) \delta J_{k, l, m}(t)\right\rangle$ is shown in Fig. S13 in SI. At $T=300 \mathrm{~K}$, both TIP4P/2005 and SWM4NDP predict a diffusion coefficient value approximately 23 and 44 times larger than that of the bulk at room temperature (exp. $D=2.30$ $\mathrm{nm}^{2} / \mathrm{ns}$ ), respectively. Near $200 \mathrm{~K}$, the diffusion coefficient in the $N=776$ droplet approaches the experimental value of supercooled water ${ }^{53}$ at $260.1 \mathrm{~K}$.

We attribute this large value relative to the bulk water to the fact that in $N=776$ droplets the $93 \%$ of the molecules are found in the subsurface $\left(411 \mathrm{H}_{2} \mathrm{O}\right.$ molecules out of 776$)$ and surface $\left(108 \mathrm{H}_{2} \mathrm{O}\right.$ molecules out of 776$)$, where molecules diffuse fast via a "jump" mechanism, which does not exist in bulk solution. The high value of diffusion coefficient is consistent with experimental suggestions that reactions in microdroplets that accelerate by orders of magnitude relative to the bulk solution may also indicate a significant increase in the value of the diffusion coefficient. ${ }^{54,55}$ The diffusion is expected to be much higher in nanoscopic droplets because of the larger surface contribution and the significant contribution of the shape fluctuations.

We find the forward rate constant $(\mathrm{CIP} \longrightarrow$ SSIP) by using the time evolution of the interionic distance of the MD trajectories. We compute the time-correlation function of a state variable $n_{\mathrm{CIP}}$ that has the value of 1 if the interionic distance is less than $3.3 \AA$ (for SWM4NDP) and 0 if it is greater than this value. The correlation function is fitted to $\phi(t)=$ $p_{\mathrm{CIP}} p_{\mathrm{SSIP}} \exp \left(-k_{f} t\right)+p_{\mathrm{CIP}} p_{\mathrm{CIP}}$, where $p_{\mathrm{CIP}}$ and $p_{\text {SSIP }}$ denote probabilities of CIP and SSIP,

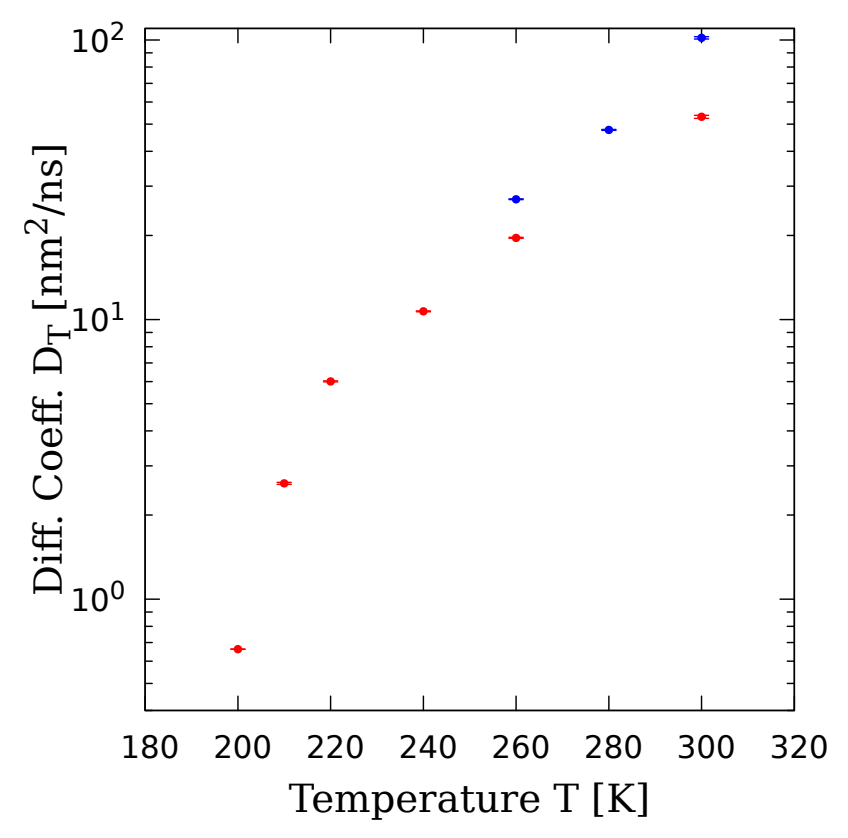

Figure 5: Diffusion coefficient of $\mathrm{H}_{2} \mathrm{O}$ as a function of temperature for $N=776$. The red points are for TIP4P/2005 and the blue for SWM4-NDP.

respectively. An example of the decay of $\left\langle n_{\mathrm{CIP}}(t) n_{\mathrm{CIP}}(0)\right\rangle$ is shown in Fig. S14 in SI and the values are presented in Table 2. The back rate constant (SSIP $\longrightarrow \mathrm{CIP}$ ) can be computed by $k_{b}=k_{f} / K_{e q}$, where $K_{e q}$ is shown in Table 1 . As it is expected, the reaction rate decreases with temperature for both models. Overall, the rate constant is higher in the TIP4P/2005 model than in SWM4-NDP. In SWM4-NDP the transitions are very few but the life-time of CIP is longer than in TIP4P/2005. The difference is attributed to the fact that in SWM4-NDP the CIP-SSIP transition occurs closer to the surface than in the TIP4P/2005. Near the surface it is expected for the CIP to live longer than in the TIP4P/2005 model because of the much lower dielectric constant in this location. The droplet electric field on the surface also may contribute in this stabilization because the ion pair is oriented with the negative site $\left(\mathrm{Cl}^{-}\right)$toward the hydrogen-site richer surface.

The dynamics of the interconversion reaction is affected by two factors that are not present in bulk solution. Firstly, the $\mathrm{Cl}^{-}$is less solvated than the $\mathrm{Na}^{+}$ion since it is found almost at the surface, therefore, it is mainly the motions 
Table 2: Self-diffusion coefficient of $\mathrm{H}_{2} \mathrm{O}$ and rate coefficient $\left(k_{f}\right)$ of the conversion of CIP to SSIP vs $T$ for TIP4P/2005 and SWM4-NDP. The values of $D$ shown in Fig. 5 are found in Table S4 in SI. As a reference, the experimental value of $D$ for water is $2.30 \mathrm{~nm}^{2} / \mathrm{ns}$ at room temperature and SWM4-NDP has been parametrized to reproduce this value $\left(2.33 \mathrm{~nm}^{2} / \mathrm{ns} \pm 0.02\right.$ from Ref. $\left.{ }^{45}\right)$. $\tau$ is the dissociation lifetime $\left(1 / k_{f}\right)$.

\begin{tabular}{|c|c|c|c|c|c|}
\hline Model & $T(\mathrm{~K})$ & $\mathrm{D}\left(\mathrm{nm}^{2} / \mathrm{ns}\right)$ & $k_{f}(1 / \mathrm{ns})$ & $\tau(\mathrm{ns})$ & error in $\tau$ \\
\hline TIP4P/2005 & 300 & 53.1 & 7.04 & 0.142 & 0.007 \\
& 260 & 19.6 & 2.34 & 0.428 & 0.012 \\
& 220 & 6.01 & 0.19 & 5.360 & 0.112 \\
\hline SWM4-NDP & 300 & 101.8 & 2.43 & 0.412 & 0.019 \\
& 280 & 47.0 & 0.64 & 1.561 & 0.048 \\
& 260 & 26.7 & 0.35 & 2.837 & 0.055 \\
\hline
\end{tabular}

of the solvent molecules around $\mathrm{Na}^{+}$that determine the dynamics. ${ }^{22}$ Secondly, the much faster diffusion of the solvent molecules in the droplet relative to the bulk solution counterpart, especially, in the subsurface and surface. These factors may decrease the coupling of the interionic distance reaction coordinate with the solvent. As a result, no diffusive barrier crossing is found in the direct monitoring of the CIP-SSIP transitions. Fig. S15 in SI shows representative trajectory segments with CIP-SSIP transitions from a trajectory at $T=260 \mathrm{~K}$ using SWM4NDP. Lack of diffusive motion at the barrier top is also found for TIP4P/2005, which shows that the weak coupling is extended in the entire subsurface. In contrast, in the bulk solution as it has been demonstrated by other authors ${ }^{38,56}$ there is a strong coupling between the solvent and the interionic distance reaction coordinate, which leads to several re-crossing at the barrier top of the free energy profile.

\section{Conclusion}

The modeling of $\mathrm{NaCl}$ ion-pairing in cold aqueous droplets showed that in colder droplets ion pairing is more likely to take place near the surface where the stability of the SSIP relative to the CIP increases. This is opposite to the results of bulk solution simulations. ${ }^{33,34,38}$ The polarizable model shows a substantial stabilization of the SSIP relative to CIP not only at low temperature but also at $T=300 \mathrm{~K}$. A sideresult of modeling via SWM4-NDP (polarizable model) is an interior broad minimum for $\mathrm{Cl}^{-}$ ions in a droplet in addition to the well-known minimum in the surface. ${ }^{51,52}$ This shows that the non-polarizable force fields overemphasize the interior local minimum.

In the study of the reaction dynamics, we found a weak coupling between the interionic $\mathrm{NaCl}$ distance reaction coordinate and the solvent degrees of freedom. The reduced coupling is mainly attributed to the presence of $\mathrm{Cl}^{-}$near the surface and to the fast diffusion of $\mathrm{H}_{2} \mathrm{O}$ molecules. In contrast to bulk solution where the crossing of the free energy barrier (along the interionic distance) is diffusive, there are no significant re-crossings in droplets. We estimated the self-diffusion of $\mathrm{H}_{2} \mathrm{O}$ by introducing a method that resolves the difficulties of employing mean-square displacement and the velocity autocorrelation function for computing the diffusion coefficient in spherical confined geometry. We found that the $\mathrm{H}_{2} \mathrm{O}$ self-diffusion coefficient at room temperature is at least an order of magnitude larger than that in bulk solution. It is at $\approx 210 \mathrm{~K}$ that the diffusion coefficient of $\mathrm{H}_{2} \mathrm{O}$ in the droplet equals the bulk value at room temperature.

Because of the shift in the location of ion distributions and ion-pairing with temperature, we propose to exploit alternating low and high temperature as a way to control the adduct formation with macromolecules in droplet-based 
ionization methods used in mass spectrometry. Bombardment of cold charged droplets with other molecules may be a possible way to remove $\mathrm{Na}^{+}$ions from the salt enriched interface. Enhanced salt release from droplets that carry the analytes may considerably extend the capability of native mass spectrometry analysis because it may allow for the salinity of the parent bulk solution of a biological sample to be closer to a physiological concentration of $\mathrm{NaCl}$ or $\mathrm{KCl}$. Protons are another major ionic species in charged droplets. Experiments and computations have detected enhanced proton activity on ice at $155 \mathrm{~K} .{ }^{58,59}$ Molecular dynamics simulations predicted that the migration of surfacehydrated protons is $\approx 2800$ slower than that of bulk protons at $190 \mathrm{~K}$ because they can be locally trapped by the undercoordinated water molecules. ${ }^{58,59}$ Thus, it was deduced that the activity detected in experiments arises from the higher proton concentration. ${ }^{58,59}$ It has also been found that protons are expelled from the ice bulk because of poor solubility in ice. ${ }^{60-62}$ The present study and accumulated evidence from the literature supports the hypothesis that protons in cold aqueous droplets will also be expelled to the surface and their migration may slow down due to local trapping. Therefore, the alternating cooling and heating of droplets may also provide a possible method of controlling the degree of protonation in proteins. We have shown that protonation and sodiation lead to different conformations of macromolecules, ${ }^{57}$ therefore, the migration of ions and their nature may also affect the conformations.

In summary, the expulsion of ions from the interior of a supercooled or ice droplet may be exploited in MS experiments in order to eliminate undesirable salts and control the charge states of biological macromolecules.

We envision that since it is possible for $\mathrm{H}_{2} \mathrm{O}$ in supercooled droplets to expel chemical species to the interface that otherwise reside in the interior at room temperature, the question of establishing a deeper relation between the solubility in supercooled $\mathrm{H}_{2} \mathrm{O}$ and ice ${ }^{63-66}$ may be addressed. We currently extend the ion-pairing study at low temperature to LiF where, differently from the $\mathrm{NaCl}$ pair, both ions show a propensity for the droplet interior at room temperature.

\section{Methods}

Here we present the main points of the computational methods. A detailed account of the methodology in found in Sec. S1 of the SI. The systems that were simulated are shown in Table S1 of the SI. We performed equilibrium molecular dynamics (MD) simulations of aqueous nanodroplets (a) with a single $\mathrm{NaCl}$ pair, (b) multiple $\mathrm{NaCl}$ pairs, and (c) charged with multiple $\mathrm{Na}^{+}$ions with a smaller number of $\mathrm{Cl}^{-}$ counterions and vice versa.

The majority of the systems were composed of $N=776 \mathrm{H}_{2} \mathrm{O}$ molecules $\left(R_{e}=1.77 \mathrm{~nm}\right)$ and fewer of $N=3000 \mathrm{H}_{2} \mathrm{O}$ molecules $\left(R_{e}=\right.$ $2.78 \mathrm{~nm})$. We selected a droplet of $N=$ $776 \mathrm{H}_{2} \mathrm{O}$ to do the majority of the computations because this size is large enough to clearly distinguish the bulk-like interior of a droplet from the subsurface and at the same time this size is computationally feasible to be studied at low temperature and with a polarizable model. The MD simulations were performed using the software NAMD v2.14 ${ }^{67}$ and the trajectories were visualized using VMD 1.9.4a47. ${ }^{68}$ The water molecules were modeled with the TIP4P/2005 (transferable intermolecular potential with four points) model ${ }^{69}$ and the SWM4-NDP model. ${ }^{45}$ The ion parameters are described in Sec. S1 of SI and for the TIP4P/2005 are shown in Table $\mathrm{S} 2$ of SI. The TIP4P/2005 simulations were performed in the temperature range $200 \mathrm{~K}$ $300 \mathrm{~K}$ and the SWM4-NDP simulations in $260 \mathrm{~K}-300 \mathrm{~K}$.

\section{Computation of diffusion coeffi- cient in droplets}

Droplets present a confining environment that restricts the range of molecule movement. The use of common formulae that relates the diffusion coefficient to the integral of the velocity correlation function or, equivalently, to the mean-squared displacement poses certain difficulties. The problem has already been pointed 
out by B. J. Berne and co-workers ${ }^{70}$ regarding the diffusion coefficient in finite-sized systems. Here we calculate the diffusion coefficient in a droplet using correlation function formalism and the macroscopic description of the diffusion in a spherical cavity.

The evolution of the molecule position is given by the Laplace equation with the Neumann boundary conditions

$$
\frac{\partial P(\mathbf{r}, t)}{\partial t}=D \nabla^{2} P(\mathbf{r}, t)
$$

where $P$ denotes probability density, and $D$ the diffusion coefficient.

The general solution of the Laplace equation is

$$
P(\mathbf{r}, t)=\sum_{\substack{k, l, m \\|m| \leqslant l}} B_{k, l, m} e^{-D t / a_{k, l}^{2}} j_{l}\left(r / a_{k, l}\right) Y_{l, m}(\theta, \phi)
$$

where $B_{k, l, m}$ are the numerical coefficients determined by the initial conditions. $r, \theta, \phi$ are the spherical polar coordinates corresponding to the molecule position $\mathbf{r}$. $Y_{l, m}(\theta, \phi)$ is the spherical harmonics of order $l$ and rank $m . j_{l}$ is the spherical Bessel function of the first kind, $a_{k, l} / R_{0}$ are the solutions of $j_{l}^{\prime}(x)=0$ imposed by the Neumann boundary conditions and $R_{0}$ the droplet's equimolar radius.

Using the orthonormality conditions for the spherical Bessel functions we arrive at the identity

$$
\begin{array}{r}
B_{k, l, m} e^{-D t / a_{k, l}^{2}}=\left\langle r^{2} j_{l}\left(r / a_{k, l}\right) Y_{l, m}^{\star}(\theta, \phi)\right\rangle= \\
\int_{r \leqslant R_{0}} d^{3} \mathbf{r} r^{2} j_{l}\left(r / a_{k, l}\right) Y_{l, m}^{\star}(\theta, \phi) P(\mathbf{r}, t)
\end{array}
$$

We introduce a microscopic quantity $\delta J_{k, l, m}$ corresponding to the decay of the probability density of the molecule location using the following identity

$$
\delta J_{k, l, m}(t)=r^{2} j_{l}\left(r_{i}(t) / a_{k, l}\right) Y_{l, m}\left(\theta_{i}(t), \phi_{i}(t)\right)
$$

for a molecule $i$.

Using the linear response ansatz ${ }^{71}$ we equate the decay of the macroscopic expectation (3) to the single molecule autocorrelation function $\frac{a_{k, l}^{2}}{D}=\frac{1}{\left\langle\delta J_{k, l, m}^{\star}(0) \delta J_{k, l, m}(0)\right\rangle} \int_{0}^{\infty} d t\left\langle\delta J_{k, l, m}^{\star}(0) \delta J_{k, l, m}(t)\right\rangle$

We use the spherical Bessel function of order $l=1$

$$
j_{1}(x)=\frac{\sin x}{x}-\frac{\cos x}{x^{2}}
$$

The first solution of the Neumann b.c. is $a_{1,1}=2.082 R_{0}$. From Eq. 5 we can find $D$ by computing the time autocorrelation function (right-hand side of 5) from the MD trajectories and by using the value of $a_{1,1}$. The program for the computation of the diffusion coefficient has been prepared by SC.

\section{Supporting Information}

(S1) Details of the computational methods and models. (S2) The trajectories of the distance of $\mathrm{Na}^{+}$and $\mathrm{Cl}^{-}$from the droplet $\mathrm{COM}$ and the interionic distance of a sole $\mathrm{NaCl}$ pair in a droplet of $N=776 \mathrm{H}_{2} \mathrm{O}$ molecules modeled with TIP4P/2005 at various temperatures. (S3) Dipole moment orientation of $\mathrm{NaCl}$. (S4) Ion probability density profiles using TIP4P/2005. (S5 \& S6) Ion probability density profiles using SWM4-NDP. (S7) Probability density profiles for droplets with $4 \mathrm{NaCl}$. (S8) Probability density profiles and discussion for highly charged droplets with counterions. (S9) Dynamics.

\section{Acknowledgments}

S.C. thanks Dr. Anatoly Malevanets, The University of Western Ontario for discussions on the stability of charged systems. V.K. acknowledges the province of Ontario and the University of Western Ontario for the Queen Elizabeth II Graduate Scholarship in Science and Technology. We acknowledge the financial support from Natural Sciences and Engineering Research Council (Canada). Computational resources were provided by Compute Canada. 


\section{References}

(1) Raab, S. A.; El-Baba, T. J.; Woodall, D. W.; Liu, W.; Liu, Y.; Baird, Z.; Hales, D. A.; Laganowsky, A.; Russell, D. H.; Clemmer, D. E. Evidence for Many Unique Solution Structures for Chymotrypsin Inhibitor 2: A Thermodynamic Perspective Derived from vT-ESI-IMS-MS Measurements. J. Am. Chem. Soc. 2020, 142, 17372-17383.

(2) Eun, H. J.; Ishiuchi, S.-i.; Baek, J. Y.; Lee, S.; Heo, J.; Fujii, M.; Kim, N. J. Cryogenic ion spectroscopy of adenine complexes containing alkali metal cations. Phys. Chem. Chem. Phys. 2021, 23, 6783-6790.

(3) Bergman, R.; Swenson, J. Dynamics of supercooled water in confined geometry. $\mathrm{Na}$ ture 2000, 403, 283-286.

(4) Richert, R. Dynamics of nanoconfined supercooled liquids. Annu. Rev. Phys. Chem. 2011, 62, 65-84.

(5) Christenson, H. K. Confinement effects on freezing and melting. J. Phys. Condens. Matter 2001, 13, R95.

(6) Alba-Simionesco, C.; Coasne, B.; Dosseh, G.; Dudziak, G.; Gubbins, K.; Radhakrishnan, R.; SliwinskaBartkowiak, M. Effects of confinement on freezing and melting. J. Phys. Condens. Matter 2006, 18, R15.

(7) Imrichová, K.; Veselỳ, L.; Gasser, T. M.; Loerting, T.; Neděla, V.; Heger, D. Vitrification and increase of basicity in between ice Ih crystals in rapidly frozen dilute $\mathrm{NaCl}$ aqueous solutions. J. Chem. Phys. 2019, 151, 014503.

(8) Cerveny, S.; Mallamace, F.; Swenson, J.; Vogel, M.; Xu, L. Confined water as model of supercooled water. Chem. Rev. 2016, 116, 7608-7625.

(9) Kaneko, T.; Bai, J.; Akimoto, T.; Francisco, J. S.; Yasuoka, K.; Zeng, X. C.
Phase behaviors of deeply supercooled bilayer water unseen in bulk water. Proc. Natl. Acad. Sci. U.S.A. 2018, 115, 4839 4844.

(10) Jiang, J.; Gao, Y.; Zhu, W.; Liu, Y.; Zhu, C.; Francisco, J. S.; Zeng, X. C. First-Principles Molecular Dynamics Simulations of the Spontaneous Freezing Transition of 2D Water in a Nanoslit. J. Am. Chem. Soc. 2021,

(11) Kaneko, T.; Bai, J.; Yasuoka, K.; Mitsutake, A.; Zeng, X. C. New computational approach to determine liquidsolid phase equilibria of water confined to slit nanopores. J. Chem. Theory Comput. 2013, 9, 3299-3310.

(12) Zhu, C.; Gao, Y.; Zhu, W.; Liu, Y.; Francisco, J. S.; Zeng, X. C. Computational prediction of novel ice phases: A perspective. J. Phys. Chem. Lett. 2020, 11, 7449 7461.

(13) Koga, K.; Tanaka, H.; Zeng, X. First-order transition in confined water between highdensity liquid and low-density amorphous phases. Nature 2000, 408, 564-567.

(14) Fischer, J. K.; Sippel, P.; Denysenko, D.; Lunkenheimer, P.; Volkmer, D.; Loidl, A. Supercooled water confined in a metal-organic framework. Communications Physics 2020, 3, 1-9.

(15) Gallo, P.; Bachler, J.; Bove, L. E.; Böhmer, R.; Camisasca, G.; Coronas, L. E.; Corti, H. R.; de Almeida Ribeiro, I.; de Koning, M.; Franzese, G. et al. Advances in the study of supercooled water. Eur Phys J E Soft Matter 2021, 44, 1-36.

(16) Muralidharan, A.; Pratt, L.; Chaudhari, M.; Rempe, S. Quasi-chemical theory for anion hydration and specific ion effects: Cl-(aq) vs. F-(aq). Chem. Phys. Lett.: $X$ 2019, 4, 100037.

(17) Zhong, J.; Kumar, M.; Anglada, J.; Martins-Costa, M.; Ruiz-Lopez, M.; 
Zeng, X. C.; Francisco, J. S. Atmospheric Spectroscopy and Photochemistry at Environmental Water Interfaces. Annu. Rev. Phys. 2019, 70, 45-69.

(18) Malek, S. M. A.; Poole, P. H.; SaikaVoivod, I. Thermodynamic and structural anomalies of water nanodroplets. Nat. Commun. 2018, 9, 2402.

(19) Malek, S. M. A.; Kwan, V.; SaikaVoivod, I.; Consta, S. Low Density Interior in Supercooled Aqueous Nanodroplets Expels Ions to the Subsurface. J. Am. Chem. Soc. 2021, 143, 13113-13123.

(20) Laria, D.; Fernández-Prini, R. Molecular dynamics study of water clusters containing ion pairs: From contact to dissociation. J. Chem. Phys. 1995, 102, 76647673 .

(21) Laria, D.; Fernández-Prini, R. Ion-pair solvation in aqueous clusters. Chem. Phys. Lett. 1993, 205, 260-266, cited By 19.

(22) Consta, S.; Kapral, R. Ionization reactions of ion complexes in mesoscopic water clusters. J. Chem. Phys. 1999, 111, 1018310191.

(23) Zidi, Z. Solvation of sodium-chloride ion pair in water cluster at atmospheric conditions: Grand canonical ensemble Monte Carlo simulation. J. Chem. Phys. 2005, 123, 064309.

(24) Mizoguchi, A.; Ohshima, Y.; Endo, Y. Microscopic hydration of the sodium chloride ion pair. J. Am. Chem. Soc. 2003, 125, 1716-1717.

(25) Hou, G.-L.; Liu, C.-W.; Li, R.-Z.; Xu, H.G.; Gao, Y. Q.; Zheng, W.-J. Emergence of Solvent-Separated $\mathrm{Na}+-\mathrm{Cl}-\mathrm{Ion}$ Pair in Salt Water: Photoelectron Spectroscopy and Theoretical Calculations. J. Phys. Chem. Lett. 2017, 8, 13-20.

(26) Knipping, E.; Lakin, M.; Foster, K.; Jungwirth, P.; Tobias, D.; Gerber, R.; Dabdub, D.; Finlayson-Pitts, B. Experiments and simulations of ion-enhanced interfacial chemistry on aqueous $\mathrm{NaCl}$ aerosols. Science 2000, 288, 301-306.

(27) Perera, L.; Berkowitz, M. L. Many-body effects in molecular dynamics simulations of $\mathrm{Na}+(\mathrm{H} 2 \mathrm{O}) \mathrm{n}$ and $\mathrm{Cl}-(\mathrm{H} 2 \mathrm{O})$ n clusters. J. Chem. Phys. 1991, 95, 1954-1963.

(28) Tandy, J.; Feng, C.; Boatwright, A.; Sarma, G.; Sadoon, A. M.; Shirley, A.; Das Neves Rodrigues, N.; Cunningham, E. M.; Yang, S.; Ellis, A. M. Communication: Infrared spectroscopy of saltwater complexes. J. Chem. Phys. 2016, $144,121103$.

(29) Heindel, J. P.; Xantheas, S. S. The ManyBody Expansion for Aqueous Systems Revisited: II. Alkali Metal and Halide Ion-Water Interactions. J. Chem. Theory Comput. 2021, 17, 2200-2216.

(30) Herman, K. M.; Heindel, J. P.; Xantheas, S. S. The many-body expansion for aqueous systems revisited: III. Hofmeister ion-water interactions. Phys. Chem. Chem. Phys. 2021, 23, 11196-11210.

(31) Partanen, L.; Mikkelä, M.-H.; Huttula, M.; Tchaplyguine, M.; Zhang, C.; Andersson, T.; Björneholm, O. Solvation at nanoscale: Alkali-halides in water clusters. J. Chem. Phys. 2013, 138, 044301.

(32) Smith, J. W.; Saykally, R. J. Soft x-ray absorption spectroscopy of liquids and solutions. Chem. Rev. 2017, 117, 1390913934.

(33) Venkateshwaran, V.; Vembanur, S.; Garde, S. Water-mediated ion-ion interactions are enhanced at the water vapor-liquid interface. Proc. Natl. Acad. Sci. U.S.A. 2014, 111, 8729-8734.

(34) Wick, C. D. $\mathrm{NaCl}$ dissociation dynamics at the air- water interface. J. Phys. Chem. C 2009, 113, 2497-2502. 
(35) Otten, D.; Onorato, R.; Michaels, R.; Goodknight, J.; Saykally, R. Strong surface adsorption of aqueous sodium nitrite as an ion pair. Chem. Phys. Lett. 2012, 519, 45-48.

(36) Hua, W.; Verreault, D.; Huang, Z.; Adams, E. M.; Allen, H. C. Cation effects on interfacial water organization of aqueous chloride solutions. I. Monovalent cations: $\mathrm{Li}+, \mathrm{Na}+, \mathrm{K}+$, and $\mathrm{NH} 4+. J$. Phys. Chem. B 2014, 118, 8433-8440.

(37) Schweighofer, K.; Benjamin, I. Ion pairing and dissociation at liquid/liquid interfaces: Molecular dynamics and continuum models. J. Chem. Phys. 2000, 112, 14741482 .

(38) Dang, L. X.; Schenter, G. K.; Wick, C. D. Rate theory of ion pairing at the water liquid-vapor interface. J. Phys. Chem. C 2017, 121, 10018-10026.

(39) Wick, C. D.; Kuo, I.-F. W.; Mundy, C. J.; Dang, L. X. The effect of polarizability for understanding the molecular structure of aqueous interfaces. J. Chem. Theory Comput. 2007, 3, 2002-2010.

(40) Luo, G.; Bu, W.; Mihaylov, M.; Kuzmenko, I.; Schlossman, M. L.; Soderholm, L. X-ray reflectivity reveals a nonmonotonic ion-density profile perpendicular to the surface of ercl3 aqueous solutions. J. Phys. Chem. $C$ 2013, 117, 19082-19090.

(41) Chakraborty, D.; Patey, G. How crystals nucleate and grow in aqueous $\mathrm{NaCl}$ solution. J. Phys. Chem. Lett. 2013, 4, 573578.

(42) Misbah, C.; Müller-Krumbhaar, H.; Temkin, D. Interface structure at large supercooling. Journal de Physique I 1991, 1, 585-601.

(43) Singh, R. S.; Palmer, J. C.; Panagiotopoulos, A. Z.; Debenedetti, P. G. Thermodynamic analysis of the stability of planar interfaces between coexisting phases and its application to supercooled water. J. Chem. Phys. 2019, 150, 224503.

(44) Niblett, S. P.; Limmer, D. T. Ion Dissociation Dynamics in an Aqueous Premelting Layer. J. Phys. Chem. B 2021, 125, 2174-2181.

(45) Lamoureux, G.; Harder, E.; Vorobyov, I. V.; Roux, B.; MacKerell, A. D. A polarizable model of water for molecular dynamics simulations of biomolecules. Chem. Phys. Lett. 2006, 418, 245-249.

(46) Gladich, I.; Roeselová, M. Comparison of selected polarizable and nonpolarizable water models in molecular dynamics simulations of ice I h. Phys. Chem. Chem. Phys. 2012, 14, 11371-11385.

(47) Muchova, E.; Gladich, I.; Picaud, S.; Hoang, P. N.; Roeselova, M. The Ice- Vapor Interface and the Melting Point of Ice I h for the Polarizable POL3 Water Model. J. Phys. Chem. A 2011, 115, 5973-5982.

(48) Wilson, M. A.; Pohorille, A. Interaction of monovalent ions with the water liquidvapor interface: A molecular dynamics study. J. Chem. Phys. 1991, 95, 60056013 .

(49) Perera, L.; Berkowitz, M. L. Structure and dynamics of Cl-(H2O) 20 clusters: The effect of the polarizability and the charge of the ion. J. Chem. Phys. 1992, 96, 8288 8294 .

(50) Werhahn, J. C.; Akase, D.; Xantheas, S. S. Universal scaling of potential energy functions describing intermolecular interactions. II. The halide-water and alkali metal-water interactions. J. Chem. Phys. 2014, 141, 064118.

(51) Caleman, C.; Hub, J. S.; van Maaren, P. J.; van der Spoel, D. Atomistic simulation of ion solvation in water explains surface preference of halides. Proc. Natl. Acad. Sci. U.S.A. 2011, 108, 6838-6842. 
(52) Burnham, C. J.; Petersen, M. K.; Day, T. J.; Iyengar, S. S.; Voth, G. A. The properties of ion-water clusters. II. Solvation structures of $\mathrm{Na}+, \mathrm{Cl}-$, and $\mathrm{H}+$ clusters as a function of temperature. $J$. Chem. Phys. 2006, 124, 024327.

(53) Price, W. S.; Ide, H.; Arata, Y. Selfdiffusion of supercooled water to $238 \mathrm{~K}$ using PGSE NMR diffusion measurements. J. Phys. Chem. A 1999, 103, 448-450.

(54) Lhee, S.; Lee, J. K.; Kang, J.; Kato, S.; Kim, S.; Zare, R. N.; Nam, H. G. Spatial localization of charged molecules by salt ions in oil-confined water microdroplets. Sci. Adv. 2020, 6, eaba0181.

(55) Wei, Z.; Li, Y.; Cooks, R. G.; Yan, X. Accelerated reaction kinetics in microdroplets: Overview and recent developments. Annu. Rev. Phys. Chem. 2020, 71, $31-51$.

(56) Ciccotti, G.; Ferrario, M.; Hynes, J. T.; Kapral, R. Dynamics of ion pair interconversion in a polar solvent. J. Chem. Phys. 1990, 93, 7137-7147.

(57) Consta, S.; In Oh, M.; Kwan, V.; Malevanets, A. Strengths and weaknesses of molecular simulations of electrosprayed droplets. J. Am. Soc. Mass Spectrom. 2018, 29, 2287-2296.

(58) Kato, F.; Sugimoto, T.; Matsumoto, Y. Direct experimental evidence for markedly enhanced surface proton activity inherent to water ice. J. Phys. Chem. Lett. 2020, 11, 2524-2529.

(59) Park, K.; Lin, W.; Paesani, F. Fast and slow proton transfer in ice: The role of the quasi-liquid layer and hydrogen-bond network. J. Phys. Chem. B 2014, 118, 80818089 .

(60) Lee, D. H.; Kang, H. Proton Transport and Related Chemical Processes of Ice. J. Phys. Chem. B 2021, 125, 8270-8281.
(61) Schwartz, A. J.; Pollack, G. H. Ice-Melting Dynamics: The Role of Protons and Interfacial Geometry. Langmuir 2017, 33, 5585-5591.

(62) Cowin, J.; Tsekouras, A.; Iedema, M.; $\mathrm{Wu}, \mathrm{K}$; Ellison, G. Immobility of protons in ice from 30 to 190 K. Nature 1999, 398, 405-407.

(63) Makkonen, L. Salinity and growth rate of ice formed by sea spray. Cold Regions Science and Technology 1987, 14, 163-171.

(64) Tsironi, I.; Schlesinger, D.; Späh, A.; Eriksson, L.; Segad, M.; Perakis, F. Brine rejection and hydrate formation upon freezing of $\mathrm{NaCl}$ aqueous solutions. Phys. Chem. Chem. Phys. 2020, 22, 7625-7632.

(65) Vrbka, L.; Jungwirth, P. Brine rejection from freezing salt solutions: A molecular dynamics study. Phys. Rev. Lett. 2005, 95, 148501 .

(66) Conde, M.; Rovere, M.; Gallo, P. Spontaneous NaCl-doped ice at seawater conditions: Focus on the mechanisms of ion inclusion. Phys. Chem. Chem. Phys. 2017, 19, 9566-9574, cited By 26.

(67) Phillips, J. C.; Braun, R.; Wang, W.; Gumbart, J.; Tajkhorshid, E.; Villa, E.; Chipot, C.; Skeel, R. D.; Kalé, L.; Schulten, K. Scalable molecular dynamics with NAMD. J. Comput. Chem. 2005, 26, 1781-1802.

(68) Humphrey, W.; Dalke, A.; Schulten, K. VMD: Visual Molecular Dynamics. J. Mol. Graphics 1996, 14, 33-38.

(69) Abascal, J. L. F.; Vega, C. A general purpose model for the condensed phases of water: TIP4P/2005. J. Chem. Phys. 2005, 123, 234505.

(70) Liu, P.; Harder, E.; Berne, B. On the calculation of diffusion coefficients in confined fluids and interfaces with an application to the liquid- vapor interface of water. J. Phys. Chem. B 2004, 108, 6595-6602. 
(71) Chandler, D. Introduction to modern statistical mechanics; New York, Oxford University Press, 1987. 